\title{
Was ist Ökonomie? Das Interdisziplinäre Studentische Kolloquium (ISK) an der Humboldt-Universität zu Berlin
}

\author{
Anja Breljak, Felix Kersting, Klaas Miersch, Timo Stieglitz und \\ Iris Wohnsiedler
}

\section{Zusammenfassung}

Das Interdisziplinäre Studentische Kolloquium (ISK) ist ein seit 2013 an der Humboldt-Universität zu Berlin stattfindendes wöchentliches Format zur kritischen Diskussion von Ökonomietheorien und anderen wissenschaftlichen Ansätzen, die sich mit ökonomischen Fragen auseinandersetzen. Das ISK verfolgt dabei drei Ziele: 1) VWL stärker als politische Ökonomie zu verstehen, 2) interdisziplinäre Zugänge zu fördern und 3) die Teilnehmerinnen und Teilnehmer zu empowern. Für die Umsetzung dieser Ziele haben sich eine offene und inklusive Atmosphäre als ebenso förderlich erwiesen wie der Ansatz des selbstbestimmten und autodidaktischen Lernens. Die dauerhafte Schaffung eines Diskussions- und Reflexionsraums nach geisteswissenschaftlichem Vorbild führte unter anderem zu weiteren studentischen Seminaren und einigen veröffentlichten Texten.

\footnotetext{
A. Breljak $(\bowtie) \cdot$ F. Kersting $\cdot$ K. Miersch · T. Stieglitz $\cdot$ I. Wohnsiedler Humboldt-Universität zu Berlin, Berlin, Deutschland

E-Mail: a.breljak@sensing-media.de

F. Kersting

E-Mail: f.kersting@hu-berlin.de

K. Miersch

E-Mail: klaas.miersch@hu-berlin.de

T. Stieglitz

E-Mail: stieglti@hu-berlin.de

I. Wohnsiedler

E-Mail: wohnsiei@hu-berlin.de
} 


\section{Schlüsselbegriffe}

Studentische Lehre · VWL-Kritik · Selbstbestimmtes Lernen · Interdisziplinarität

\section{$1 \quad$ Entstehungskontext}

Die Gründung des Interdisziplinären Studentischen Kolloquiums (ISK) an der Humboldt-Universität zu Berlin hatte eine Art Momentum: Im Sommer 2013 war die sogenannte Eurokrise auf ihrem Höhepunkt angekommen und mit ihr die Diskussionen um die Rolle der Wirtschaftswissenschaften für das Aufkommen dieser Wirtschafts- und Finanzkrise. Zeitgleich entstanden in Deutschland, in Europa und vielen anderen Regionen der Welt Gruppierungen wie Rethinking Economics und das Netzwerk Plurale Ökonomik, die sich kritisch mit den Wirtschaftswissenschaften auseinandersetzten und deren neoklassische Annahmen und Theorien hinterfragten. Die Gründung des ISK war geprägt von diesen Umständen.

Wichtig für die Gründung waren allerdings auch andere Entwicklungen: Dazu gehörte erstens die Veränderung der Hochschulen durch den EU-weiten Bologna-Prozess. So viele Schattenseiten die Vereinheitlichung der Studiengänge auf Bachelor- und Masterniveau auch hat, sind durch die Etablierung von Kombinations-Bachelorstudiengängen und die Erleichterung des Hochschuloder Fachwechsels (etwa zwischen Bachelor und Master) auch die Wissensflüsse zwischen den Disziplinen noch fester verankert worden (Klemperer et al. 2002). So war das ISK von Beginn an, wenn auch an der wirtschaftswissenschaftlichen Fakultät angesiedelt, neben der Ökonomik auch von Disziplinen wie der Psychologie, den Kulturwissenschaften, der Philosophie, den Sozialwissenschaften, der Physik oder der Mathematik geprägt.

Zweitens spielte auch die Geschichte der Studierendenkämpfe an der Humboldt-Universität (HU) eine zentrale Rolle: Die Forderungen der Studierenden nach mehr Autonomie und Mitbestimmung in der Lehre führten dazu, dass 1992 unter anderem das Format des Projekttutoriums eingeführt wurde. Damit wurde eine zweisemestrige Veranstaltung, die von Studierenden in Eigenregie geplant und durchgeführt werden konnte, in die Semesterpläne aufgenommen und für das Studium anrechenbar gemacht, inklusive Vergütung der Verantwortlichen. Auf diese Weise sollten einerseits ,blinde Flecken' in der Lehre ausgeglichen, andererseits Studierende auch materiell dazu befähigt werden, die 
Lehre mitzugestalten. ${ }^{1}$ Das ISK ging aus einem solchen Projekttutorium hervor: Zwei Studierende, die kurz vor ihrem Bachelor-Abschluss in Philosophie und VWL standen, stellten im Februar 2011 einen Antrag auf ein Projekttutorium mit dem Titel: „Was ist Ökonomie? Theorie und Kritik des ökonomischen Denkens“. Der Titel der Veranstaltung bezog sich auf Andreas Jägers Werk Was ist Ökonomie? Zur Formulierung eines wissenschaftlichen Problems im 19. Jahrhundert (Jäger 1999). Diese titelgebende Frage, was Ökonomie eigentlich sei, die Jäger in die Anfänge der systematischen Wirtschaftswissenschaften geführt hatte, wurde schließlich auch zum Leitmotiv derjenigen Gruppe, die im Zuge der zwei Semester Projekttutorium entstand und im Rahmen des ISK ihre Diskussionen über Ökonomietheorien weiterführen wollte. Daraus entwickelte sich schließlich auch die Studierendeninitiative „Was ist Ökonomie?“, die neben anderen Aktivitäten im Bereich der kritischen Wirtschaftswissenschaften die organisatorische Plattform für das ISK darstellt.

Drittens war die spezielle Geschichte der wirtschaftswissenschaftlichen Fakultät der HU entscheidend für die Gründung sowohl der Studierendeninitiative als auch des ISK. Die Humboldt-Universität stand im Ostteil Berlins und war damit eine Hochschule der DDR, in der der Marxismus-Leninismus gelehrt wurde. Das galt auch für die wirtschaftswissenschaftliche Fakultät. Nach der Wende ging es der Universität ebenso wie der Fakultät darum, diese Geschichte möglichst rasch abzuschütteln: Das Personal der wirtschaftswissenschaftlichen Fakultät wurde Anfang der 1990er Jahre fast vollständig durch neue Mitarbeiterinnen und Mitarbeiter sowie Professorinnen und Professoren aus den westdeutschen Bundesländern ersetzt. Das Ziel war eine „,wissenschaftliche“ (und darunter verstanden die Verantwortlichen vor allem: mathematische) VWL, die sich an der neoklassischen Theorieschule orientierte (Düppe 2015). ${ }^{2}$ Eine Dekade später war die VWL an der HU Berlin deutlich an einem rigiden Mainstream ausgerichtet. So hat etwa das Masterprogramm VWL laut Studiengangsbeschreibung eine „quantitative Ausrichtung" mit „theoretischer Fundierung"; man versteht sich

\footnotetext{
${ }^{1}$ Siehe die Informationen zu Projekttutorien (Online: https://www.hu-berlin.de/de/studium/ reform/projekttutorien/wasisteinpt, [12.01.2020]) und deren Ausrichtung an der HumboldtUniversität zu Berlin.

${ }^{2}$ Für eine umfangreiche Dokumentation der Geschichte der Wirtschaftswissenschaftlichen Fakultät an der Humboldt-Universität zu Berlin siehe ihre Dokumentation (Online: http:// hicks.wiwi.hu-berlin.de/history/ [12.01.2020]).
} 
„als ,hartes ' Fach““3 Auch lehnt ein führender Vertreter der Fakultät Forderungen nach einer Quote für plurale Lehrstühle grundsätzlich ab. ${ }^{4}$ Die Fakultät legt großen Wert auf Wettbewerb zwischen den Studierenden (dazu passt, dass alle Notenschnitte öffentlich einsehbar sind) sowie auf ein repräsentatives Gebäude und setzt auf eine fragwürdige Nähe zu Unternehmen, die hier bis dato offen für sich werben und Stiftungsprofessuren finanzieren. ${ }^{5}$ Studierenden, die etwas verändern wollten oder auch nur kritische Fragen an ihr Fach hatten, wurde überwiegend mit Härte und Unverständnis begegnet. Der daraus resultierende Frust machte sich im Projekttutorium bemerkbar und stiftete Energien des Trotzes und der Rebellion. Hinzu kam, dass jeder noch so kleine administrative Akt, sei es eine Raumbuchung oder die Aufhängung eines Plakates, zum Streitfall mit der Verwaltung wurde und die Unzufriedenheit weiter nährte. Im Zuge des zweiten Semesters des Projekttutoriums wurde klar: Die Teilnehmenden wollen diesen Zustand und diese Art der Fachkultur nicht länger hinnehmen. Sie wollen kritische Fragen stellen, Texte lesen, nachdenken können, sie wollen Annahmen hinterfragen, die Geschichte der Wirtschaftswissenschaften kennenlernen, feministische Theorien diskutieren und sich aktiv in die Lehre einbringen. Mit dem Ende des Projekttutoriums im Wintersemester 2012/2013 war dieses Anliegen noch nicht an sein Ziel gekommen, es gründete sich die Gruppe „Was ist Ökonomie?“” und es folgte die Veranstaltungsankündigung für das erste Interdisziplinäre Studentische Kolloquium (ISK) im Sommersemester 2013.

\footnotetext{
${ }^{3}$ Siehe z. B. die Studiengangsbeschreibung für das Masterprogramm in VWL (Online: https://www.wiwi.hu-berlin.de/de/studium/sb/studium/Master\%20VWL.html [12.01.2020]).

${ }^{4}$ Siehe z. B. dieses Interview mit Michael Burda in der taz (Online: https://taz.de/Volkswirtschaftslehre-in-der-Kritik/!5084130/. [20.05.2020].

${ }^{5}$ Z. B. eine „SAP-Stiftungsprofessur für Organisationsökonomik- Zukunft der Arbeit“ und eine „Lakestar-Stiftungsprofessur für Venture Capital“. Für weitere Informationen zu den Professuren an der Wirtschaftswissenschaftlichen Fakultät der Humboldt-Universität zu Berlin siehe nachfolgende Übersicht (Online: https://www.wiwi.hu-berlin.de/de/ professuren [12.01.2020]).
} 


\section{Innovative Lösung im Sinne einer pluralen, sozioökonomischen Hochschullehre}

Das ISK war von Beginn an als wöchentliches Kolloquium konzipiert, in dem ein offener Diskussionsraum entstehen sollte. Dabei stand die gemeinsame Lektüre im Vordergrund, ebenso wie die Interessen der Teilnehmenden sowie aktuelle politische und ökonomische Themen. War das vorangegangene Projekttutorium noch als „Berufsbezogene Zusatzqualifikation“ mit drei ECTS Punkten anrechenbar gewesen, weil es als Lehrformat von der Universität abgedeckt wurde, handelte es sich beim ISK um eine selbstorganisierte Veranstaltung, die vom Engagement der Teilnehmenden lebte und deren Initiative brauchte.

Auch wenn es anfänglichen Widerstand seitens der Fakultät gab, gelang es, das ISK im Gebäude der Wirtschaftswissenschaftlichen Fakultät stattfinden zu lassen. Dass es dort abgehalten werden konnte und kann, ist der bis heute währenden Unterstützung durch einen Professor zu verdanken. Diese wichtige Allianz war es, die auch viele andere Vorhaben an der Fakultät ermöglichte. $\mathrm{Zu}$ Beginn wäre eine darüber hinausgehende Institutionalisierung des ISK vonseiten der wirtschaftswissenschaftlichen Fakultät, wo Lehrveranstaltungen als Qualifizierungen verstanden werden und das bewertete Endergebnis im Fokus steht, undenkbar gewesen. Über die Semester und Jahre hinweg gewöhnte sich die Fakultät an das ISK. Der Möglichkeit einer Institutionalisierung stand allerdings auch später das Selbstverständnis des ISK im Weg: Als offenes Diskussions- und Reflexionsformat sollte es vor allem dem Prinzip des selbstbestimmten und autodidaktischen Lernens folgen. Eine Bewertung von ,Leistungen“ war dafür nicht zielführend, weil es in erster Linie um Empowerment und Selbstbildung gehen sollte. Zudem sind Prüfungsleistungen und Teilnahmebescheinigungen auch Teil jenes Wettbewerbsdrucks, den auch Studierende anderer Universitäten vielfach beklagen, wie etwa die Untersuchung von Engartner und Schweitzer-Krah (2019) zeigt. Im ISK sollte stattdessen die Diskussion auf Augenhöhe im Vordergrund stehen. Das manifestiert sich auch darin, dass das ISK jedes Semester von einem anderen Team organisiert wird, welches sich idealerweise aus dem Teilnehmendenkreis des vorherigen Semesters zusammensetzt.

Das Interdisziplinäre Studentische Kolloquium (ISK) ist als studentischselbstorganisierter, disziplinär nicht zugeordneter und offener Gesprächs- und Diskussionsraum ohne feste Form konzipiert. Als Vorbild dienten dafür wissenschaftliche Kolloquien, wie sie in der Philosophie und den Kulturwissenschaften schon lange üblich sind, wo die text- oder vortragsbasierte Diskussion im Vordergrund steht. Dabei interagieren die Teilnehmenden auf Augenhöhe, die 
Tab. 1 Oberthemen im ISK, 2013-2019. (Eine Liste mit allen Texten findet sich auf der Homepage der „Was ist Ökonomie“-Gruppe (Online: https://wasistoekonomie.de/ wordpress/wp-content/uploads/ISK-Texte.pdf [12.01.2020]).)

\begin{tabular}{l|l}
\hline Semester & Oberthema \\
\hline Sommer 2013 & Wissenschaftstheorie der VWL \\
\hline Winter 2013/2014 & Zugänge zu Ökonomie \\
\hline Sommer 2014 & Zugänge zu Ökonomie \& Gastvorträge \\
\hline Winter 2014/2015 & Ungleichheit \\
\hline Sommer 2015 & Vorstellung eigener Arbeiten \\
\hline Winter 2015/2016 & Schulden \& Vorstellung eigener Arbeiten \\
\hline Sommer 2016 & Zugänge zu Ökonomie \\
\hline Winter 2016/2017 & Neoliberalismus \\
\hline Sommer 2017 & Feminismus und Ökonomie \\
\hline Winter 2017/2018 & Globalisierung \& Vorstellung eigener Arbeiten \\
\hline Sommer 2018 & Methodologie der VWL \\
\hline Winter 2018/2019 & Arbeit \\
\hline Sommer 2019 & Politische Ökonomie \\
\hline Winter 2019/2020 & Wert \\
\hline
\end{tabular}

Diskussion wird idealerweise von der Gruppe selbst getragen und es wird ein gemeinsames Erkenntnisinteresse verfolgt. Das Erkenntnisinteresse des ISK war und ist es, kritische Perspektiven auf ökonomische Theorien und Fragestellungen zu entwickeln und aus anderen Disziplinen kommende Zugänge kennenzulernen, um so Raum für Reflexion zu schaffen.

Das ISK hat mit diesem ,formfreien Format' drei Ziele im Blick: Erstens sollen darin die Wirtschaftswissenschaften als politische Ökonomie gedacht werden, d. h. es geht darum, Theorien und Ansätze zu erkunden, die es erlauben, Ökonomie als Wissenschaft wie auch als Studium zu politisieren und im gesellschaftlichen Kontext zu verorten. So spielten dafür Untersuchungen, die nach der Wirkmacht ökonomischen Wissens in der Gesellschaft oder nach den normativen Aspekten ökonomischer Theorie und Methodik fragen, eine zentrale Rolle. Aber auch die Wissenschaftstheorie der VWL, jüngere Arbeiten zu Ungleichheit, Schulden und Arbeit und die Geschichte des Neoliberalismus und sein Verhältnis zu ökonomischer Theoriebildung waren wichtige Themen und Theoriefelder, die im ISK diskutiert wurden. Damit orientiert sich das ISK auch 
in der Auswahl der Themen entlang der Kriterien für sozio-ökonomische Bildung, wie Hedtke (2015) sie vorgeschlagen hat: Demnach stehen nämlich „Wirtschaft und Wirtschaften in der Gesellschaft“ (ebd., S. 27) als Gegenstand und die „Kontextualisierung des Denkens und Handelns in der und über die Wirtschaft“ (ebd.) als Ziel im Vordergrund dieses bildungspolitischen Ansatzes. Dies spiegelt sich in den bisher bearbeiteten Themen wider, die in Tab. 1 aufgelistet sind.

Zweitens hat das ISK zum Ziel, interdisziplinäre Ansätze zusammenzubringen. Dahinter steht die Überzeugung, dass benachbarte Disziplinen nicht nur spannende, sondern mitunter auch deutlich kritischere Forschung und Reflexion zur Ökonomie bieten können. Im Gegensatz zur Vision der sozio-ökonomischen Bildung (Hedtke 2015) umfasst die Interdisziplinarität des ISK nicht nur die Sozialwissenschaften, sondern im Besonderen auch geisteswissenschaftliche Fächer wie die Kultur-, Medien- und Literaturwissenschaften, Philosophie und Anthropologie. Einerseits trägt das ISK damit den Studienhintergründen der Teilnehmerinnen und Teilnehmer Rechnung, die vielfach geisteswissenschaftliche Neben- oder Hauptfächer studierten. Andererseits ist das Format des Kolloquiums ein vor allem in den Geistes- und Kulturwissenschaften kultivierter Gesprächs- und Diskussionsraum, der hier als Vorbild diente. Im Zentrum steht dabei das, was Moldaschl (2015, S. 259 ff.) als Kompetenz des „reflexiven Theoriegebrauchs" bezeichnet hat. Denn geistes- und kulturwissenschaftliche Mittel und Werkzeuge erlauben nicht nur, Prämissen, Metaphern und Paradigmen systematisch zu hinterfragen, sie befördern im besten Fall auch das kritische Denken und die Fähigkeit, Diversitäten und auch Unvereinbarkeiten verschiedener Theorieströmungen auszuhalten.

Drittens ist das Ziel des ISK, die Teilnehmerinnen und Teilnehmer zu empowern. Das gemeinsame Diskutieren und Durchdenken komplexer Gedanken verschiedenster Ansätze, Annahmen und Modelle gibt den Teilnehmerinnen und Teilnehmern ein gutes Fundament, um ihre Fragen auch in wirtschaftswissenschaftlichen Vorlesungen, auf Konferenzen und anderen Veranstaltungen stellen zu können. Mit dem Prinzip des selbstbestimmten und autodidaktischen Lernens soll so ganz im Sinne von Freire (1970) die Konsumhaltung der Vorlesungssituation nicht nur im ISK, sondern auch in Vorlesungen, die nach wie vor das wichtigste Vermittlungselement wirtschaftswissenschaftlicher Lehre sind, aufgebrochen werden. Durch die Verbindung des ISK mit der Studierendeninitiative „Was ist Ökonomie?“ stand und steht den Teilnehmerinnen und Teilnehmern außerdem eine aktivistische Plattform zur Verfügung, in der sie gemeinsam mit anderen Gedanken, Texte oder Projektideen entwickeln und verwirklichen können. 
Insgesamt zielt das ISK darauf ab, einen Raum zu schaffen, in dem die Inhalte des Ökonomiestudiums reflektiert und neue Perspektiven diskutiert werden können. Das traditionelle geisteswissenschaftliche Format des Kolloquiums wird vor allem vor dem Hintergrund starrer Denk- und Lehrstrukturen innerhalb des klassischen VWL-Studiums (Bäuerle et al. 2020) zu einer innovativen Lösung. Denn einerseits markiert das ISK damit nicht nur thematisch einen deutlichen Unterschied zu den monodisziplinär aufgestellten Wirtschaftswissenschaften, sondern auch zu ihren didaktischen Mitteln. Andererseits befördert das ISK das kritische Hinterfragen und die Fähigkeiten des Argumentierens gegen eine institutionell verankerte Wissenschaftspraxis und ihre Autoritäten.

Zur Verwirklichung dieser drei Ziele (Politisierung, Interdisziplinarität und Empowerment) hat sich eine offene und inklusive Atmosphäre als besonders förderlich erwiesen. Das Prinzip des selbstbestimmten Lernens in einer Gruppe setzt nämlich voraus, dass Themen- und Verfahrensvorschläge von den Teilnehmenden selbst kommen, dass neuen Teilnehmenden möglichst von Beginn an Gehör geschenkt wird und dass sie frühzeitig in die Selbstorganisation des Kolloquiums eingebunden werden. Weil das ISK auch zur Präsentation eigener Arbeiten oder zum gemeinsamen Lesen eines Werkes im Stile eines Lesezirkels genutzt werden kann, gibt es kein Patentrezept für die Durchführung, sondern vor allem eine Reihe von handlungsleitenden Regeln zur Umsetzung der offenen Atmosphäre: So dient die Sitzordnung im Kreis oder in der U-Form zur Schaffung eines gemeinsamen Raumes, in dem sich alle Teilnehmenden sehen können. Zu Beginn des Semesters oder bei Neueinstieg bekommen alle die Gelegenheit zur Selbstvorstellung. Der Semesterplan kann und sollte an die Wünsche der Beteiligten auch im Laufe des Semesters angepasst werden. Anstehende organisatorische oder vorbereitende Aufgaben werden von Sitzung zu Sitzung verteilt. Eine oder mehrere Moderatorinnen und Moderatoren achten auf eine ausgeglichene Redeliste. Am Ende des Semesters findet sich möglichst aus dem Kreis der Teilnehmenden eine Gruppe, die das nachfolgende Semester plant und organisiert.

Die Arbeitsmethoden in den Sitzungen selbst sind variabel und unterliegen der Abstimmung der jeweils an den Sitzungen teilnehmenden Studierenden. Für die Wahl der Methoden zur Textdiskussion beispielsweise spielt neben dem Text (Form, disziplinärer Hintergrund, zusätzlicher Materialbedarf) auch die Zahl der Anwesenden, die meistens zwischen sechs und zwölf liegt, eine Rolle. Üblicherweise finden sich eine oder mehrere Personen, die den Text kurz zusammenfassen, Thesen ausarbeiten oder diskussionswürdige Punkte herausstellen. Als besonders hilfreich hat sich für die Diskussion das sogenannte ,Blitzlicht` erwiesen: Vor den einführenden Bemerkungen zum Text gibt es für 
alle die Gelegenheit, Eindrücke zum Text zu schildern, Verständnisfragen zu stellen oder eigene Thesen in die Diskussion zu geben. Damit können sich erste Schwerpunkte für die spätere Diskussion herauskristallisieren, was den Verlauf einer Sitzung inhaltlich strukturiert und thematisch ausgewogener macht. Dieses Verfahren ermöglicht es, von Anfang an alle einzubeziehen und anfängliches Schweigen aufzulösen.

Im Hintergrund dieser Überlegungen zur Lern-Atmosphäre steht eine Problematik, die dem Format des Kolloquiums geschuldet ist und nicht unterschlagen werden sollte: Das ISK als studentisch-selbstbestimmter Gesprächs- und Diskussionsraum setzt voraus, dass die Zahl der Teilnehmenden überschaubar bleibt, damit ein Gespräch unter möglichst vielen stattfinden kann. Denn je größer die Zahl der Teilnehmenden, desto schwieriger gestaltet sich die Möglichkeit der niedrigschwelligen Beteiligung an der Diskussion. Insofern eignet sich ein derartiges Kolloquium nicht als Einführungsveranstaltung, etwa um einer großen Zahl von Studienanfängerinnen und Studienanfängern einen Einblick in die ,kritische VWL' zu geben. Das Format lebt sogar von einer gewissen Durchmischung jener, die schon mehrere Semester teilnehmen und jenen, die neu dazukommen.

Andererseits droht gerade durch die Kontinuität der Teilnahme das Entstehen einer zunehmenden Wissenskluft: Denn je länger Teilnehmende im ISK mitmachen, desto größer wird das Wissens- und Methodengefälle zwischen ihnen und neu einsteigenden Studierenden. Schließlich sind die Fähigkeiten und Fertigkeiten einen Text zu lesen, zu verstehen und zu diskutieren, Argumente herauszustellen und eloquent zu vertreten, etwas Erlernbares. Die Teilnehmenden des ISK lernen im Zuge ihrer Teilnahme eben diese, an der wirtschaftswissenschaftlichen Fakultät wenig gepflegten Fertigkeiten und entwickeln ihr Text- und Leseverständnis, ihren Überblick über Forschungs- und Theorielandschaften und ihre Urteilsfähigkeit in Bezug auf Argumentationen weiter. Jene, die neu einsteigen, sind nicht immer auf dieses Gefälle gefasst. Umso wichtiger ist die aktive Inklusion neuer Teilnehmerinnen und Teilnehmer durch die ,alten Hasen' in das ISK.

\section{$3 \quad$ Wirkungen}

Das ISK hat im Laufe der Jahre seines Bestehens vielen Studierenden die Möglichkeit geboten, sich gemeinsam mit anderen über alternative Lern- und Lehrinhalte auseinanderzusetzen. Welche Wirkungen hatte das, wie hat das so Gelernte die Organisatorinnen und Organisatoren, die Teilnehmerinnen und Teil- 
nehmer sowie die wirtschaftswissenschaftliche Fakultät der Humboldt-Universität beeinflusst?

Zunächst ist zu bemerken, dass sich das ISK verstetigt hat. Durch die aktive Inklusion von Neueinsteigerinnen und Neueinsteigern sind nicht nur starke Bindungen zwischen den Teilnehmenden entstanden. Aus neuen Teilnehmenden sind so auch recht schnell neue Organisatorinnen und Organisatoren des ISK geworden. So besteht die Veranstaltung weiterhin (Stand: Winter 2019), obwohl inzwischen kein Gründungsmitglied mehr aktiv an der Gestaltung des ISK beteiligt ist.

Eine repräsentative Erhebung der Wirkungen des ISK hat nie stattgefunden. Zwar wurde die Veranstaltung jedes Semester evaluiert, dies wurde aber nicht systematisch dokumentiert. Bei einer nicht repräsentativen Befragung von ehemaligen und aktiven Teilnehmenden für diesen Beitrag gaben mehrere Menschen an, dass das ISK ihr Denken und ihren ,Blick auf die Welt" nachhaltig geprägt habe. Mehrere Teilnehmende sagten aus, dass das Kolloquium ihre Überzeugung genährt hat, dass wirtschaftswissenschaftliche Diskurse stärker normative und politische Fragestellungen in den Blick nehmen sollten. Zudem gaben viele an, dass das ISK ihren Blick auf die produktive Rolle der Geistes- und Kulturwissenschaften in der Diskussion ökonomischer Fragen gerichtet habe. Mehrere ehemalige Studierende sagten außerdem aus, dass das ISK ihnen geholfen hat, das VWL-Studium überhaupt „durchzuziehen“, weil sie sich dadurch mit anderen Studierenden, die ähnlich frustriert waren oder kritische Reflexion vermissten, solidarisieren konnten. Der Kontakt mit über den neoklassischen Kanon hinausgehenden ökonomischen Ideen hat für jene, die sich nach dem VWL-Studium für eine Promotion entschieden haben, auch zur Konsequenz gehabt, sich mit progressiveren Methoden und polit-ökonomischen Themen auseinanderzusetzen. Ein ehemaliger Student der Physik, der das Kolloquium über viele Jahre aktiv mitgestaltete, ist überzeugt, dass das Kolloquium die Wahl seines Promotionsthemas und seine akademische Laufbahn maßgeblich beeinflusst hat und weiterhin beeinflusst.

Das ISK hatte den problemorientierten Austausch zwischen Studierenden der VWL und Studierenden der Geistes-, Kultur- und Sozialwissenschaften im Fokus und konnte damit auch eine räumliche Kluft zwischen den Fakultäten der Humboldt-Universität überbrücken. Dabei haben nicht nur die VWLStudierenden von den Studierenden der Geistes-, Kultur- und Sozialwissenschaften durch neue Perspektiven und methodische Öffnungen profitiert. Auch umgekehrt ist ein besseres Verständnis für die eigene(n) Disziplin(en), ihre Rolle im akademischen Betrieb und bis dahin nicht erschlossene Anwendungsfelder deutlich geworden. 
An der Wirtschaftswissenschaftlichen Fakultät ist das ISK mittlerweile fest etabliert. Anfängliche Konflikte um Raumbuchung und Plakatierung gehören nicht mehr zur Realität des ISK, vielmehr gehört das ISK nun zur Realität der Fakultät. So ist das ISK wie selbstverständlich Teil des Vorlesungsverzeichnisses der Wirtschaftswissenschaften geworden. Das Lehrpersonal kennt das ISK mittlerweile und bietet mitunter sogar die Möglichkeit an, in Vorlesungen darauf hinzuweisen. Auch gab es seitens der Fakultät Angebote zur Institutionalisierung des ISK, die allerdings ausgeschlagen wurden.

War das Projekttutorium einstmals der Wegbereiter für das ISK, so ist das ISK selbst wiederum Wegbereiter für eine Vielzahl weiterer Projekttutorien und Q-Tutorien ${ }^{6}$ geworden. Diese studentischen Lehrformate an der HumboldtUniversität zu Berlin wurden über die Jahre hinweg von einzelnen Mitgliedern der Gruppe in Teams oder alleine immer wieder an verschiedenen Fakultäten angeboten. In den Titeln dieser studentischen Tutorien deutet sich auch an, wie stark die interdisziplinäre Verknüpfung nach wie vor ist:

- „Ökonomie und Literatur“.

- ,Wir brauchen Alternativen! Ökonomie in der Kritik.“

- „Die Berliner Start-Up Szene und der Neue Geist des Kapitalismus.“

- „Wirtschaftliches Handeln zwischen Wettbewerb und Verantwortung.“

- „Spekulationsblasen in einer interdisziplinären Betrachtung - Von der Great Depression bis zur Finanzkrise.“

- „Macht Ökonomie Gesellschaft? Zum Verhältnis von Wirtschaftswissenschaft und Gesellschaft."

- „Ökologie und Feminismus.“

Das ISK war und ist sowohl inhaltlich als auch personell ein wichtiges Sprungbrett für derart nachfolgende Veranstaltungsformate. Inhaltlich haben sich viele der studentischen Nachfolge-Tutorien auf die andiskutierten Themen des ISK gestützt, die dann in einem solchen Tutorium vertieft behandelt werden konnten. Durch die Kontinuität der Teilnahme am ISK sammelte sich über die Jahre außerdem viel Know-How über das Verfassen von Anträgen oder Organisieren von Veranstaltungsreihen, das die Teilnehmenden in der Gruppe weitergeben

\footnotetext{
${ }^{6}$ Informationen zu den Projekttutorien an der Humboldt-Universität zu Berlin finden sich online (https://www.hu-berlin.de/de/studium/reform/projekttutorien [12.01.2020]), ebenso zu Q-Tutorien (Online: https://bolognalab.hu-berlin.de/de/projekte-des-bologna.labs/qprogramm/q-tutorien [12.01.2020]).
} 
konnten. Das ISK half Studierenden, ihre Interessen und Stärken zu erkunden, Gleichgesinnte kennenzulernen, sich gegenseitig zu ermutigen - kurzum: sich gemeinsam zu empowern.

Wiewohl das ISK keine große Zahl von Menschen erreicht hat (durchschnittlich zehn Studierende pro Semester), haben die zahlreichen Projekt- und Q-Tutorien, die daraus entstanden sind, dazu geführt, dass die Zahl derjenigen Studierenden, die an der Humboldt-Universität mit kritischen, pluralen und interdisziplinären Perspektiven auf Ökonomie konfrontiert waren, deutlich höher liegt. Hinzu kommt, dass das ISK Teilnehmende dazu motiviert hat, gemeinsam Texte und Artikel zu verfassen. So ist etwa aus dem ISK im Wintersemester 2014/2015, welches sich mit dem Thema Ungleichheit auseinandergesetzt hat, ein Artikel zu Thomas Pikettys „Capital in the 21st Century“ (Piketty 2013) und dessen Rezeption in verschiedenen Theorieschulen der VWL entstanden (Fauser et al. 2016). Die vielfache Beschäftigung mit Fragen der Wirkmacht und gesellschaftlichen Rolle der VWL führte zu einem Sammelbandtext (Breljak und Kersting 2019). Die Lektüre einiger Bücher (z. B. Mirowskis „Never Let a Serious Crisis Go to Waste“ (Mirowski 2013) oder Graebers „Bullshit Jobs“ (Graeber 2018)) mündete in Rezensionen ${ }^{7}$ für die Wirtschaftszeitung Oxi. Das ISK hat sich damit als Quelle verschiedenster Effekte erwiesen: Es hat die Denkweisen der Teilnehmerinnen und Teilnehmer geprägt und so ihre Fähigkeit zum kritischen Hinterfragen von ökonomischen Theorien und Modellen, gerade auch in Gestalt einer entsprechend selbstbewussten Präsenz dieser Studierenden im universitären Lehrbetrieb, gefördert. Zudem hat das ISK sich und die studentische Lehre verstetigt und vervielfältigt, sowohl in Form von anderen Lehrveranstaltungen (siehe obige Liste von studentischen Tutorien) als auch in Form von Texten. Das Format des Kolloquiums erweist sich gerade im Kontext des VWLStudiums als produktiver Raum für kritisches (Weiter-)Denken und Schreiben.

Wie stark das ISK vom Antagonismus zum Feld der Mainstream-VWL profitiert hat, wie sehr also die Zukunft des ISK in einer möglicherweise (und wünschenswerten!) pluraleren Ökonomik denkbar ist, ist eine Frage, die gerade die Gründungsgeschichte des ISK nahelegt. Es wird darauf ankommen, auch in Zukunft Studierende aller Disziplinen dafür begeistern zu können, nicht nur ihre Unzufriedenheit über das Curriculum in Engagement für plurale Lehre zu verwandeln, sondern vor allem auch den ,Geist ${ }^{*}$ der Geisteswissenschaften, der sich im Kolloquium verbirgt, für ihr Lernen und Verstehen von ökonomischen Fragen stark zu machen.

${ }^{7}$ Online: https://oxiblog.de/author/was-ist-oekonomie/ [12.01.2020]. 


\section{Literatur}

Bäuerle, L., Pühringer, S., \& Ötsch, W. O. (2020). Wirtschaft(lich) studieren. Erfahrungsräume von Studierenden der Wirtschaftswissenschaften. Wiesbaden: Springer.

Breljak, A., \& Kersting, F. (2019). Macht Ökonomie Gesellschaft? Zur Wirkmacht ökonomischen Wissens. In D. J. Petersen, D. Willers, E. M. Schmitt, R. Birnbaum, J. H. E. Meyerhoff, S. Gießler, \& B. Roth (Hrsg.), Perspektiven einer pluralen Ökonomik (S. 43-65). Wiesbaden: Springer.

Düppe, T. (2015). Border cases between autonomy and relevance: Economic sciences in Berlin. A natural experiment. Studies in History and Philosophy of Science, 51, 22-32.

Engartner, T., \& Schweitzer-Krah, E. (2019). Wie denken Studierende über die Pluralismusdebatte in der Volkswirtschaftslehre? Ergebnisse einer quantitativen Befragung an deutschen Hochschulen. FGW-Studie Neues Ökonomisches Denken 10. Düsseldorf: Forschungsinstitut für Gesellschaftliche Weiterentwicklung (FGW). http://www. fgw-nrw.de/fileadmin/user_upload/FGW-Studie-NOED-10-Engartner-2019_01_22komplett-web.pdf. Zugegriffen: 12. Jan. 2020.

Fauser, H., Kersting, F., Müller-Hansen, F., \& Sacharow, A. (2016). Missing bridges: A pluralist analysis of the debate on Capital in the Twenty-First Century. International Journal of Pluralism and Economics Education, 7, 300-322.

Freire, P. (1970). Pedagogy of the oppressed. London: Bloomsbury Academic.

Graeber, D. (2018). Bullshit jobs: A THEORY. London: Allen Lane.

Hedtke, R. (2015). Sozioökonomische Bildung als Innovation durch Tradition. $G W$ Unterricht, 140, 18-38.

Jäger, A. (1999). Was ist Ökonomie? Zur Formulierung eines wissenschaftlichen Problems im 19. Jahrhundert. Marburg: Metropolis.

Klemperer, A., van der Wend, M., \& Witte, J. (2002). Die Einführung von Bachelor- und Master-Programmen an deutschen Hochschulen. Bonn: Deutscher Akademischer Austauschdienst.

Mirowski, P. (2013). Never Let a Serious Crisis Go to Waste. How Neoliberalism Survived the Financial Meltdown. London: Verso.

Moldaschl, M. (2015). Paradigmatisches Lernen. Oder: Wie lehrt man Sozioökonomik?. In R. Hedtke (Hrsg.), Was ist und wozu Sozioökonomie? (S. 339-369). Wiesbaden: Springer.

Piketty, T. (2013). Capital in the 21st Century. Cambridge (MA): Harvard University Press. 
Open Access Dieses Kapitel wird unter der Creative Commons Namensnennung 4.0 International Lizenz (http://creativecommons.org/licenses/by/4.0/deed.de) veröffentlicht, welche die Nutzung, Vervielfältigung, Bearbeitung, Verbreitung und Wiedergabe in jeglichem Medium und Format erlaubt, sofern Sie den/die ursprünglichen Autor(en) und die Quelle ordnungsgemäß nennen, einen Link zur Creative Commons Lizenz beifügen und angeben, ob Änderungen vorgenommen wurden.

Die in diesem Kapitel enthaltenen Bilder und sonstiges Drittmaterial unterliegen ebenfalls der genannten Creative Commons Lizenz, sofern sich aus der Abbildungslegende nichts anderes ergibt. Sofern das betreffende Material nicht unter der genannten Creative Commons Lizenz steht und die betreffende Handlung nicht nach gesetzlichen Vorschriften erlaubt ist, ist für die oben aufgeführten Weiterverwendungen des Materials die Einwilligung des jeweiligen Rechteinhabers einzuholen.

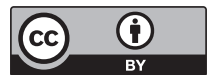

